\title{
Structural hierarchy in DNA hydrogels
}

\section{Abstract}

Sol-gel transition in aqueous DNA solutions was probed to determine the gelation time $t_{\text {gel }}$ and temperature $T_{\text {gel }}$. It was remarkable to observe that $T_{\text {gel }}$ increased from 36 to $57^{\circ} \mathrm{C}$ and tgel linearly decreased from 700 to $350 \mathrm{~s}$ with increase in DNA concentration. The hydrogels were studied by using small angle neutron scattering (SANS) $([\mathrm{DNA}] \leq 3 \%(\mathrm{w} / \mathrm{v}))$ to determine the underlying hierarchy of length scales. From structure factor profile analysis, three distinct signatures were obtained:

a. Radius of gyration $\operatorname{Rg} \approx 40 \pm 3 \mathrm{~nm}$, which assigned a length of $140 \mathrm{~nm}$ to DNA strand

b. Geometry of scattering moiety defined by the value of $\alpha \approx 2.2 \pm 0.1$ indicating Gaussian chain behavior

c. Correlation length, $\xi$ increased from 0.50 to $3.30 \mathrm{~nm}$ and the inter-chain spacing $\mathrm{d}_{0}$ decreased from 15.6 to $9.8 \mathrm{~nm}$, with increase in DNA concentration.

Physical networks were formed for concentration, $c^{*} \geq 2 \%(\mathrm{w} / \mathrm{v})$ and the system involved at least five identifiable length scales. A revisit has shown that hierarchical structure of DNA hydrogel owes its origin to considerable self-organization at the molecular level dependent on biopolymer concentration.

Keywords: DNA hydrogels, neutron scattering, guinier-regime, power-law, broad peak region, correlation length, self-assembly

\author{
Volume 2 Issue 4 - 2017
}

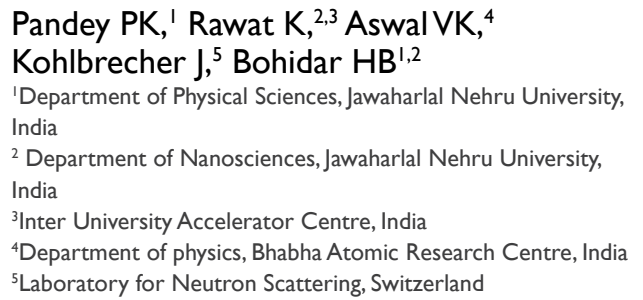

Correspondence: Bohidar HB, School of Physical Sciences, Jawaharlal Nehru University, New Delhi I I0067, India, Fax +9| || 2674 |837, Tel +9| || 26704637,

Email bohi0700@mail.jnu.ac.in

Rawat K, Inter University Accelerator Centre, India, Fax +91 । 2674 |837, Tel +9| || 26704637, Email kamla.jnu@gmail.com

Received: December 28, 2016 | Published: March 10, 2017
Abbreviations: SANS, small angle neutron scattering; EGDE, ethylene glycol diglycidyl ether

\section{Introduction}

DNA is found to be an extraordinary biopolymer because it can be used as molecular tool to construct many other objects such as nano devices, periodic arrays and genetic engineering templates. DNA plays a vital role in many biological processes like gene therapy. But due to its large extended chains it cannot be delivered directly into the cells. ${ }^{1}$ DNA hydrogels are eco-friendly because these are biocompatible and biodegradable. Unlike other hydrogels, DNA hydrogel are different in terms of efficiency and strong branched cross-linking. ${ }^{2}$ The benefit of it is one can achieve gelling under physiological conditions that allows for the encapsulation of drugs, proteins and mammalian cells in the sol phase instead of drug-loading step and denaturing conditions. ${ }^{3}$ A strongly interactive PVA and DNA cryogel was prepared and it showed a good mechanical resistance due to its heterogeneous porous structure. ${ }^{4}$ For natural DNA, formaldehyde and metal compounds such as arsenic, chromate and nickel are widely used as cross-linkers which are known carcinogens. ${ }^{5,6}$ DNA-cross-linked PAA mgel could be used to release nano particles for potential drug-delivery vehicles in response to right stimulus. ${ }^{7}$ DNA hydrogels reversibly respond to thermal stimulus, by switching between the gel and sol state (transition temperature) and enzymes when restriction sites are inserted into one of the building blocks. ${ }^{8}$

DNA hydrogels have a wide range of biomedical application in tissue engineering and drug-delivery system. ${ }^{9}$ Lee et al inspired by silkworm and spiders gave the evidence for the formation of DNA hydrogel fiber with self-entanglement prepared in the presence of ionic liquid. ${ }^{10}$ Moran et al. ${ }^{9}$ prepared DNA gel particles and studied swelling, dissolution behavior and DNA release. ${ }^{9}$ They made DNA gel particles in the presence surfactant CTAB and protein lyzozyme, but without using any cross-linker or organic solvents. Orakdogen et al. ${ }^{11}$ showed evidence of strain hardening in both physical and chemical DNA gels. ${ }^{11}$ Physical gels were prepared by physical cross-linking of DNA with heating-cooling cycle, while chemical gels were prepared by adding a cross linker like EGDE (ethylene glycol diglycidyl ether). Visco elastic properties of DNA gels and the crossover frequency for DNA in solutions that vary with concentration have been reported. ${ }^{12,13}$ Liu et al. ${ }^{14}$ described the relaxation time of double and single stranded DNA on the basis of viscosity measurement in an oscillatory flow and by stress relaxation measurements. ${ }^{14}$ DNA hydrogel relaxation dynamics has been found to be hierarchical in concentration. ${ }^{15}$ The differential shrinking of hydrogels in presence of $\mathrm{NaBr}$ and cationic surfactant has been reported. ${ }^{16}$ Rhelogical behavior of DNA hydrogels indicated that storage modulus G' and loss modulus G" are frequency independent which is due to dominance of viscoelastic relaxation of the networks at lower frequencies. ${ }^{17}$

Hierarchical self-assembly in DNA gels is the primary factor which distinguishes and makes it versatile. At appropriate concentration and controlled flexibility, one-pot self-assembly of DNA gives rise to very small nanometer range tetrahedral, dedecahedra and buckyball size complex structures. Therefore, self-assembly of DNA allows formation of complex 3-D structures. ${ }^{18}$ DNA gels exhibit universal scaled stiffening behavior that can be reproduced by a wormlike chain model keeping in mind the entropic elasticity of DNA strands. ${ }^{11}$ For tissue engineering applications, due to biocompatibility and permeability of hydrogel, one can design the required threedimensional constructions. ${ }^{19}$ In spite of the availability of a large volume of literature on DNA, the micro-structural probing of their hydrogels by SANS remains poorly explored. In this report, we have comprehensively studied DNA hydrogels using SANS to develop a general understanding of hierarchical self-organization of these networks. Considering the importance of the nucleic acids in genetic engineering and pharmaceutics, these results are of importance. 


\section{Materials and methods}

The salmon testis dsDNA sodium salt $(2000 \mathrm{bp}$, molecular weight $1.3 \times 10^{6} \mathrm{~g} / \mathrm{mol}$ ) and $\mathrm{D}_{2} \mathrm{O}$ (base solvent) were purchased from SigmaAldrich, USA. In the medium of $(0.15 \mathrm{M})$ sodium chloride and $0.015 \mathrm{M}$ sodium citrate, it has $41.2 \% \mathrm{G}-\mathrm{C}$ content and $87^{\circ} \mathrm{C}$ melting temperature. DNA was dissolved in $\mathrm{D}_{2} \mathrm{O}$ in different amounts (1$3 \%(\mathrm{w} / \mathrm{v}))$ at $60^{\circ} \mathrm{C}$, followed by heating to $90^{\circ} \mathrm{C}$ under stirring to denature the biopolymer. Finally, samples were gradually cooled to room temperature $\left(25^{\circ} \mathrm{C}\right)$ while their viscosity was measured in real time (viscometer model SV-50, A \& D Company, Japan) and the gels were subjected to SANS studies. Rigid gels were formed for DNA concentration, $c^{*} \geq 2 \%(w / v)$. The SANS experiments were carried out using the SINQ neutron source at PSI, Villegen, Switzerland. These measurements were carried out with $1.6 \mathrm{~nm}$ neutron wavelength where the scattering wave vector (q) was scanned in the range of 0.03$3.4 \mathrm{~nm}^{-1} .{ }^{20}$ The scattering profile, $\mathrm{I}(\mathrm{q})$ vs $\mathrm{q}$, exhibited characteristic signature pertaining to the internal micro-structure of the samples. All the measurements were performed using a 2D position sensitive detector of size $(96 \mathrm{~cm} \mathrm{x} 96 \mathrm{~cm})$, source wavelength of $0.8 \mathrm{~nm}$, sample to detector distance of 28 and $20 \mathrm{~m}$ and path length of quartz cuvette of
$2 \mathrm{~mm}$. All concentrations are in (w/v) unless otherwise stated.

\section{Results and discussion}

\section{Sol-gel Transition}

DNA solutions prepared with different DNA concentrations were analyzed for their characteristic sol-gel transition behavior. Time dependent evolution of viscosity $\eta$ is shown by Figure 1 for different samples. The time dependent growth of viscosity could be described by the scaling relation given by. ${ }^{21}$

$$
\eta_{r}=\varepsilon^{-k} ; t \rightarrow t_{\text {gel }}
$$

Here, relative viscosity $\eta_{r}=\frac{\eta}{\eta_{0}}, \eta_{0}$ is solvent viscosity, $\varepsilon=\left(1-\frac{\mathrm{t}}{\mathrm{t}_{\mathrm{gel}}}\right)$ and $\mathrm{t}_{\mathrm{gel}}$ is gelation time.

\section{Figure I}
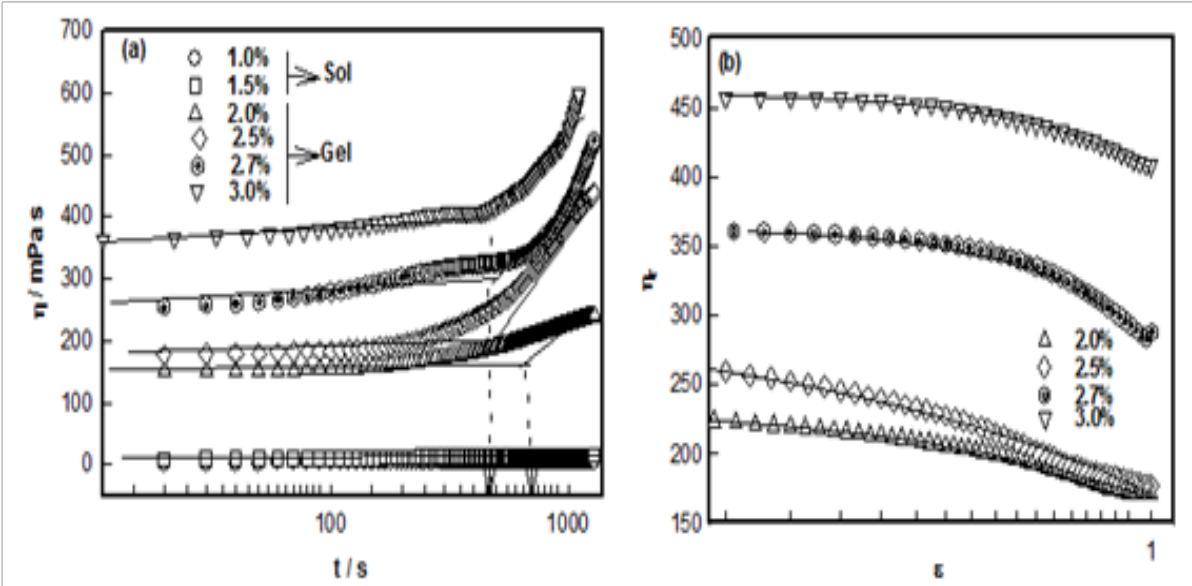

A. TEMPORAL dependence of viscosity. The arrows indicate gelation time $t_{\text {gel }}$ B. Relative viscosity $\eta_{p}$, as function of reduced time $\varepsilon=\left(1-\frac{t}{t_{g e l}}\right)$. Solid lines are fitting of data to Equation (I).

The plot between $\eta_{\mathrm{r}}$ vs. $\mathrm{t}$ shown in Figure 1A, provided the characteristic time $t_{\text {gel }}$ obtained from the change in slope which was found to decrease with DNA concentration. It was found that as the DNA concentration was increased from 1 to $3 \%$, the viscosity of DNA sol increased gradually due to the formation of inter connected physical networks. To fit relative viscosity data to time dependent power-law function was used and corresponding power-law exponent $\mathrm{k}$ was determined. Theoretical value for Rouse dynamics corresponds to $\mathrm{k}=0.7$ for conducting and $\mathrm{k}=1.3$ for percolating gel network. ${ }^{22,23} \mathrm{In}$ our case, $\mathrm{k}=0.13 \pm 0.02$, implying invariance of the network growth process with DNA content. Although it was an indicative description of gelation, but on the basis of experimental data, it was possible to give a reasonable description to gelation kinetics. The gelation time was $\mathrm{t}_{\text {gel }} \approx 700 \mathrm{~s}$ for $2 \%$ gel, which reduced to $350 \mathrm{~s}$ when the DNA concentration was $3 \%$. This clearly indicated that at higher concentration due to the propensity of DNA strands the physical entanglement was highly favorable that caused earlier gelation (Figure 2).

No gelation transition was noticed when the biopolymer concentration was less than $2 \%$. The temperature of gelation was $36^{\circ} \mathrm{C}$ for $2 \%$ sample which enhanced to $57^{\circ} \mathrm{C}$ for $3 \%$ DNA gels. It is remarkable to note that just by raising the concentration it was possible to increase the gelation temperature by $63 \%$. At the same time the gelation time reduced by almost $50 \%$. Further, the data shown in Figure 3, could be fitted to a straight line (chi-squared $=0.99$ ) with a slope of $\approx 23\left({ }^{\circ} \mathrm{C}(\mathrm{v} / \mathrm{w})\right)$. The linear dependence of gelation temperature with DNA concentration was not reported hither to. This result will have significant bearing on the applications of DNA hydrogels.

\section{Static structure factor}

The static structure factor profiles obtained from different DNA hydrogel samples are shown in Figure 4. The raw data was examined 
by checking their $1 / \mathrm{I}(\mathrm{q})$ vs $\mathrm{q}^{2}$ and $\frac{1}{\sqrt{q}}$ vs $\mathrm{q}^{2}$ behavior which correspond to Ornstein-Zernike ${ }^{24}$ and Debye-Bueche functions. ${ }^{25}$ The statistical accuracy of this fitting was poor. The best fit to the data was found when it was fitted to an empirical function given by equation (2). In this formalism, we have identified three ranges of scattering vectors q:

I. Low q-range $\left(0.05 \mathrm{~nm}^{-1} \leq \mathrm{q} \leq 0.11 \mathrm{~nm}^{-1}\right)$ was fitted to Guinier function given by

$$
\mathrm{I}(\mathrm{q}) \sim \mathrm{I}_{\mathrm{G}} \exp \left(\frac{-\mathrm{R}_{\mathrm{gq}}^{2} \mathrm{q}^{2}}{3}\right)
$$

where $\mathrm{R}_{\mathrm{g}}$ is known as radius of gyration of scattering moiety.

II. In the intermediate $\mathrm{q}$-range $\left(0.12 \mathrm{~nm}^{-1} \leq \mathrm{q} \leq 0.21 \mathrm{~nm}^{-1}\right)$, power-law function was used for data fitting $\mathrm{I}_{\mathrm{PL}}(\mathrm{q}) \sim \mathrm{q}^{-\alpha}$ where $\alpha$ is related to the geometry of scattering moiety and

In the high q-range $\left(\mathrm{q} \geq 0.22 \mathrm{~nm}^{-1}\right)$ the broad peak was fitted to a modified Lorentzian function given by, $\mathrm{I}(\mathrm{q})=\frac{I_{B P}}{\left(\mathfrak{i}+\left(\mathrm{q}-\mathrm{q}_{0}\right)\right)^{\mathrm{m}}}$ where $\mathrm{q}_{0}$ is related to peak position of scattered peak and $\xi$ is correlation length for polymer chain and $\mathrm{m}$ is a fitting parameter (Figure 4). Thus, the structure factor profile could be described by

$$
I(q)=\mathrm{I}_{\mathrm{G}} \exp \left(\frac{-\mathrm{R}_{\mathrm{gq}}^{2}}{3}\right)+I_{P L} q^{-\alpha}+\frac{\mathrm{I}_{\mathrm{BP}}}{\left(\mathfrak{1}+\left(\mathrm{q}-\mathrm{q}_{0}\right)\right)^{\mathrm{m}}}
$$

This mode of analysis yielded excellent chi-squared values $(>0.97)$ in all the cases. The cross-over between low and intermediate and intermediate and large- $\mathrm{q}$ values are identified as $\mathrm{q}^{*}$ and $\mathrm{q}^{* *}$, respectively. These defined the signature length scales given by $L^{*}\left(=\frac{2 \Pi}{\mathrm{q}^{*}}\right)$ and $L^{* *}\left(=\frac{2 \Pi}{\mathrm{q}}\right)$ which plotted for different hydrogel samples. This biopolymer had an overlap concentration of $c^{*}(\geq 2 \%)$ above which the solution was in gel state (Figure 5). Remarkably, the value of $L^{*}(=50 \mathrm{~nm})$ coincides with the persistence length of DNA. ${ }^{26,27}$ The $\mathrm{L}^{* *}$ value was $26 \mathrm{~nm}$ in the sol and about $22 \mathrm{~nm}$ in gel state (Figure $5)$. Thus, to a very good approximation, $L^{*} \approx 2 L^{* *}$. In the low q-region $\left(q<3 R_{g}^{-1}\right)$ the scattering factor was approximated by Guinierregime.

$$
I(q)=\mathrm{I}_{\mathrm{G}} \exp \left(\frac{-\mathrm{R}_{\mathrm{gq}}^{2} \mathrm{q}^{2}}{3}\right)
$$

where $I_{G}$ is the structure factor at very low $q$ and the fitting yielded value of radius of gyration $\mathrm{R}_{\mathrm{g}} \approx 40 \pm 3 \mathrm{~nm}$ in the sol state independent of DNA concentration (Figure 6). According to scaling laws correlation length dependence of $\xi$ with concentration is given as. ${ }^{28}$

$$
\hat{\imath}=R_{g}\left(\frac{c}{c^{*}}\right) \frac{v}{(1-3 v)}
$$

Where $\mathrm{c}^{*}(\approx 2 \%)$ is the typical overlap concentration and $v$ is excluded volume exponent of the system. From this scaling relation, using $\xi=0.5-3.3 \mathrm{~nm}$ (deduced from equation 6 ), $\mathrm{R}_{\mathrm{g}}=40 \mathrm{~nm}$ and $\mathrm{c}=2 \%$ and scaling exponent obtained was $v \sim 0.40-0.45$. Thus, in the sol state DNA strands were in the poor solvent environment, which changed to marginal solvent situation as the gelation temperature was reached. Therefore, the sol-gel transition was concomitant with change in the solvent quality for this biopolymer. Since, this change was in the favorable direction of solubility, there was no phase separation, but a clear transition to gel phase occurred due to a propensity in the physical entanglement of the DNA strands. We use equation (4) with caution because it strictly applies to chemically cross-linked networks and gels. In the intermediate q-regime $\left(0.12 \mathrm{~nm}^{-1} \leq \mathrm{q} \leq 0.21 \mathrm{~nm}^{-1}\right)$, powerlaw dependence of structure factor was defined by

$$
I_{P L}(q) \sim q^{-\alpha}
$$

(5)Figure

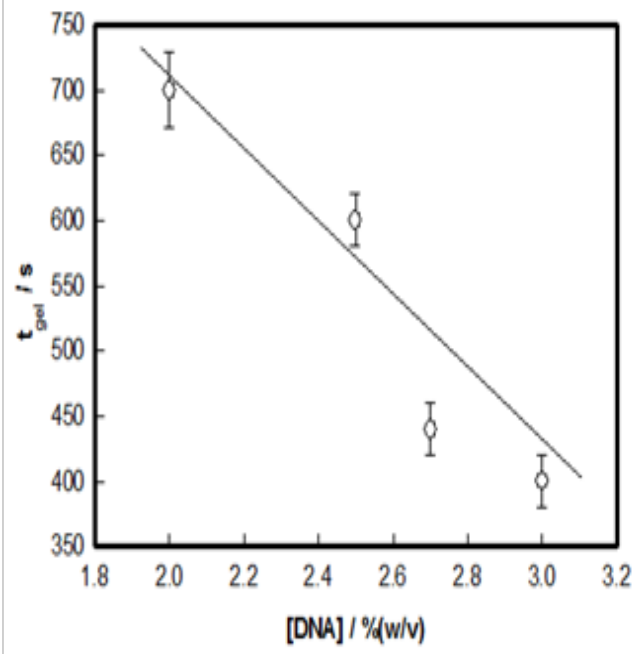

2 Variation of time of gelation $t_{\text {gel }}$ as function of DNA concentration.

The least-squares fitting of data yielded the value for $\alpha \approx 2.2 \pm 0.1$ independent of DNA concentration (Figure 6B), which attributed Gaussian chain behavior (theoretical value $=2$ ) to these strands. SANS data in the high q-region was described by a signature broad-peak, a profile normally noticed in scattering from amorphous soft materials. In this formalism, the typical distance between scattering heterogeneities is explained by d-spacing, $d_{0}$ which is the peak position of the broad correlation peak located at $\mathrm{q}_{0}$. The $\mathrm{q}_{0}$ value signifies the peak position of different morphologies such as in lamellar, cylindrical, spherical or for bi-continuous structures. ${ }^{29}$ Here in the case of hydrogels one can see the significance of such a model. We identify $\mathrm{d}_{0}$ as average distance between the polymer-poor and polymer-rich regions. The following functional form (modified Lorentzian function) described the broad peak feature seen in our system Peak position $\mathrm{q}_{0}$ is related to $\mathrm{d}_{0}$ through the relation 

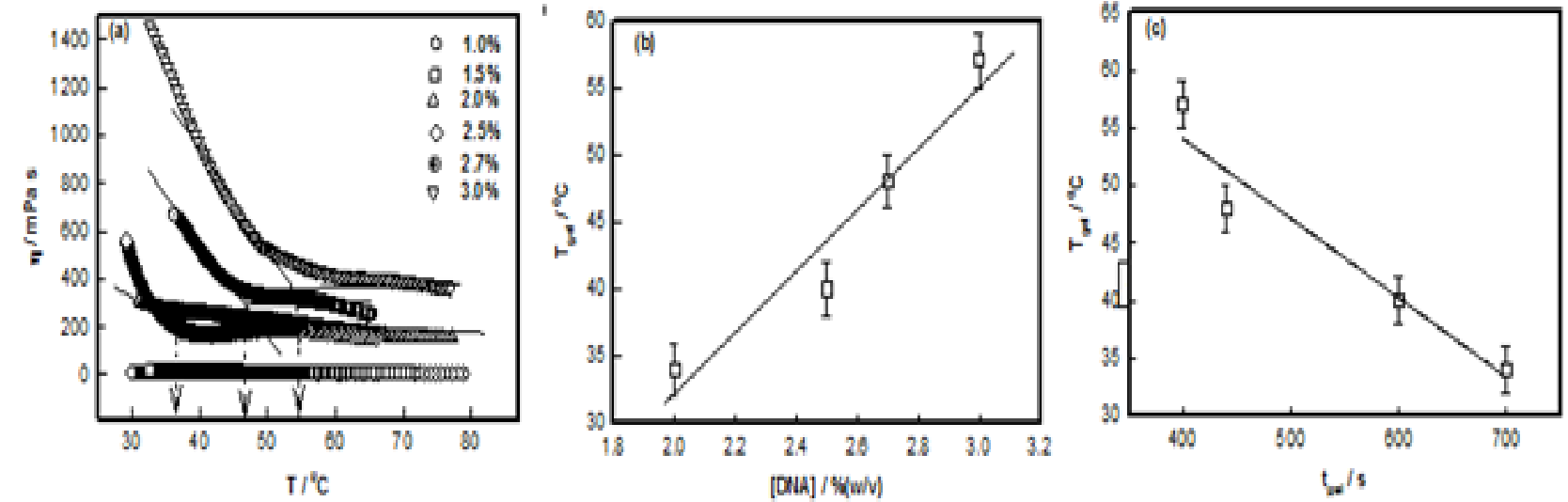

Figure 3

A. Variation of viscosity with temperature shown for different DNA concentrations.

B. Variation of gelation temperature $\mathrm{T}_{\text {gel }}$ with DNA concentrations and

C. Temporal temperature dependence. Note the Newtonian cooling rate $0.069^{\circ} \mathrm{C} / \mathrm{s}$.

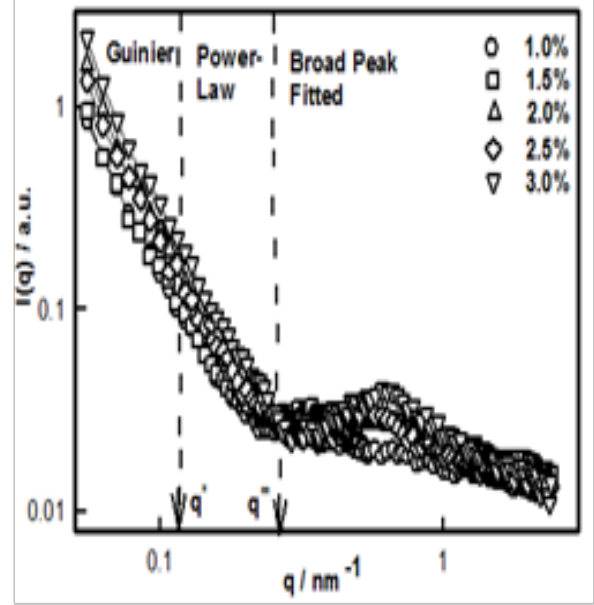

Figure 4 SANS correlation between static structure factor I(q) and scattering vector q for different DNA concentrations. These correlations are best fitted with Guinier, Power-Law and Broad peak (Lorentzian) functions in different q-ranges.
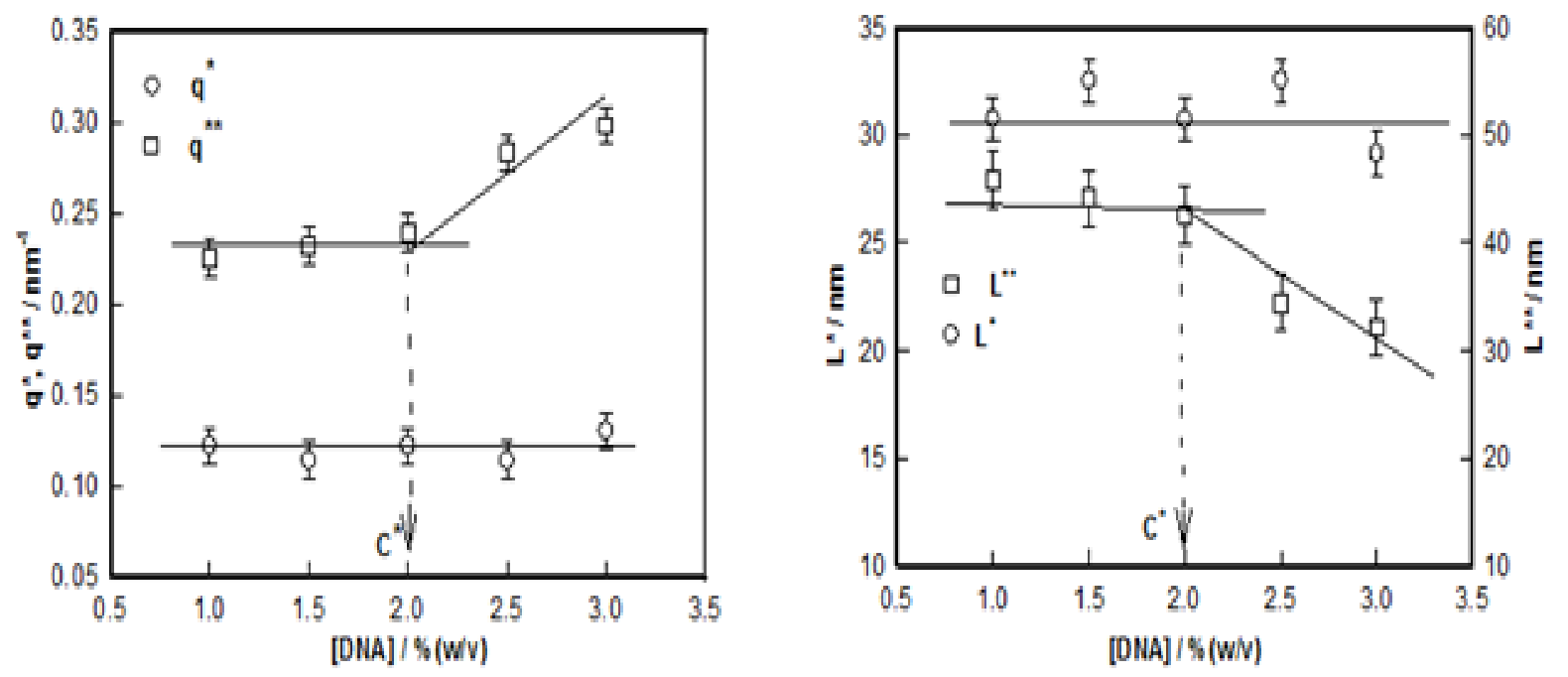

Figure 5

a. Variation of $\mathrm{q}^{*}$ and $\mathrm{q}^{* *}$ with different DNA concentrations.

b. Variation of $\mathrm{L}^{*}$ and $\mathrm{L}^{* *}$ with different DNA concentrations. Overlap concentration $\mathrm{c}^{*}$ is clearly identified here (arrow). 

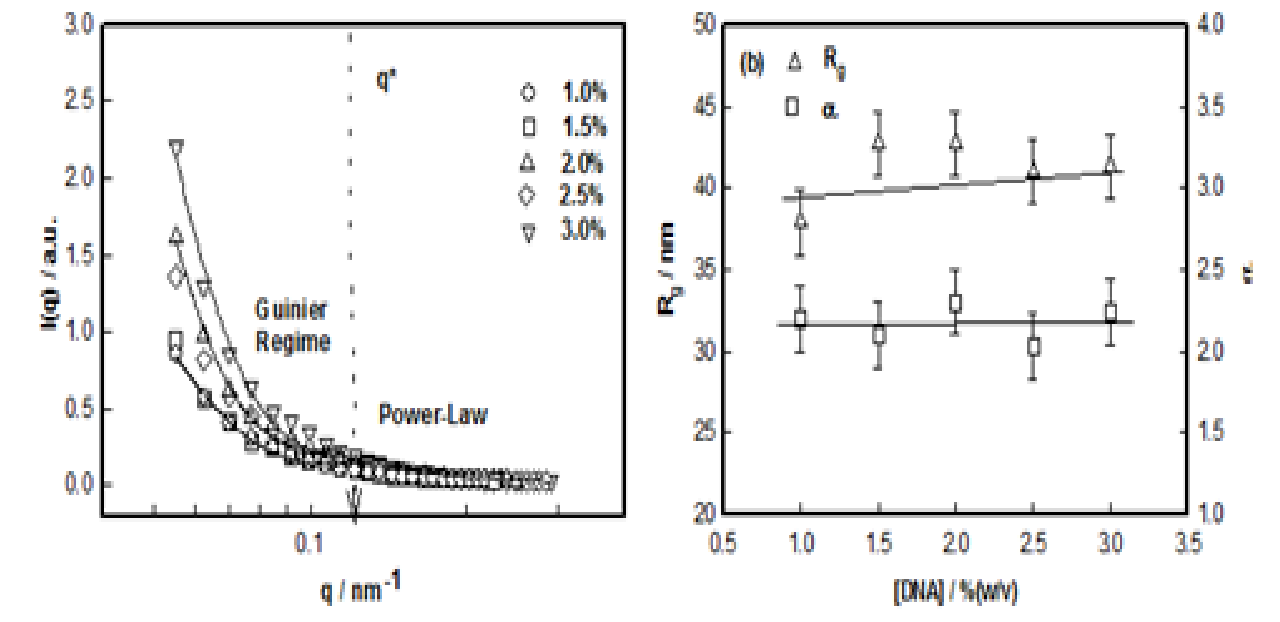

Figure 6

A. Correlation for I(q) versus $\mathrm{q}$ fitted by Guinier and Power-Law functions for different DNA concentrations at room temperature.

B. Variation of radius of gyration and geometrical scattering factor $\alpha$ with different DNA concentration. Arrow demarcates the two regions.

$$
d_{0}=\frac{2 \Pi}{q_{0}}
$$

Figure 7 data clearly shows that for the sample with $1 \%$ DNA concentration there was no correlation peak. As we increased the DNA concentration, the existence of broad peak became more prominent and its position shifted to lower- $\mathrm{q}_{0}$ value. Thus the value for $\mathrm{d}_{0}$ decreased from $16.0 \pm 1.0$ to $10.0 \pm 0.8 \mathrm{~nm}$ which signified that the hydrogel system had become compact and dense. Incidentally, the value of the exponent $m \approx 2 \pm 0.2$. Secondly, we found that with increase in DNA concentration (1 to 3\%), the correlation length, $\xi$ increased from 0.50 to $3.3 \mathrm{~nm}$. It can be inferred that as DNA concentration increased there was formation of network structures due to physical entanglement of DNA strands, which was predominant at higher DNA concentrations. This corresponded to dominant scattering arising from these structures. This behavior is depicted in Figure 8, which, in addition, defines the overlap concentration $\mathrm{c}^{*}$. Based on the SANS data, a schematic sol-gel phase diagram can be constructed which is shown in Figure 9. In the sol state, the mean inter strand distance was $\mathrm{d}_{0}=16 \pm 1 \mathrm{~nm}, \mathrm{R}_{\mathrm{g}}=40 \pm 2 \mathrm{~nm}$ and correlation length (distance of minimum approach) $\xi=0.5 \pm 0.04 \mathrm{~nm}$. These values changed to $\mathrm{d}_{0}=10.0 \pm 1.0 \mathrm{~nm}$ and $\xi=3.3 \pm 0.4 \mathrm{~nm}$ at the highest concentration used

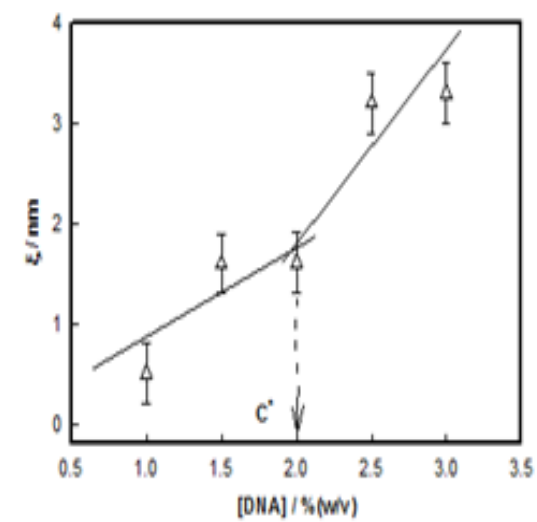

in this study. Thus, it was clearly seen that the self-assembly leading to the gelation involved at least five length scales. The other three parameters showed strong concentration dependence.

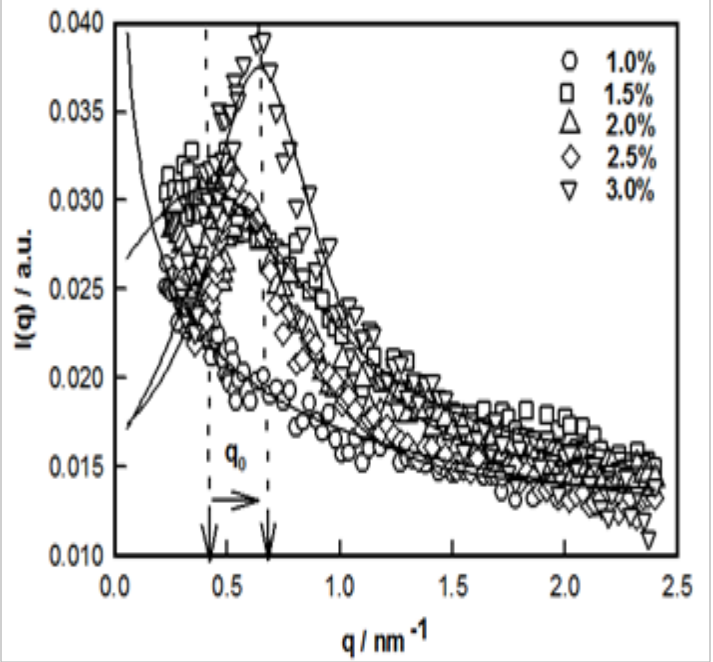

Figure 7 Correlation between I (q) versus $q$ obtained from SANS measurement for high $q$ regime performed on different DNA concentrations at room temperature. Note the red-shift of $\mathrm{q}_{0}$ (arrows).

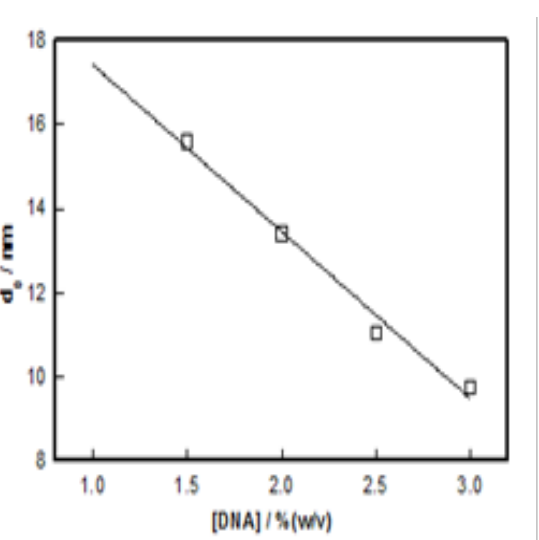

Figure 8 Variation of A. Correlation length with different DNA concentration.

B. D- spacing with different DNA concentrations. 


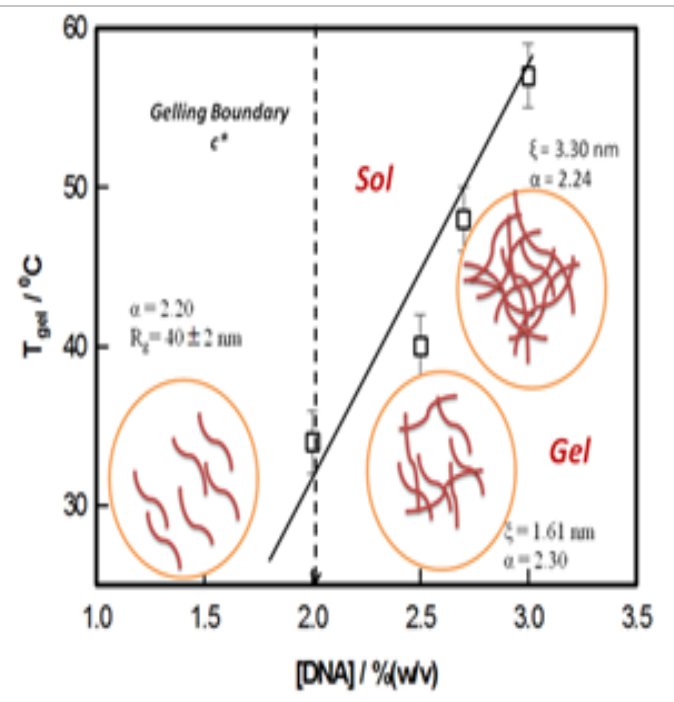

Figure 9 Schematic of sol- gel transition in DNA solution.

\section{Conclusion}

It was found that self-assembly of DNA strands precedes gelation transition. It was possible to extract micro-structural information about DNA strands with SANS measurement. The structure factor profile, I(q) vs q data, was divided into three different q-regions and were treated with specific fitting functions: $\begin{gathered}\text { low-q } \\ \text { (Guinier- regime) }\end{gathered} \quad\left(0.05 \mathrm{~nm}^{-1} \leq \mathrm{q} \leq 0.11 \mathrm{~nm}^{-1}\right), \quad \mathrm{I}(\mathrm{q})=\mathrm{I}_{\mathrm{G}} \exp \left(\frac{-\mathrm{Rg}_{\mathrm{g}}^{2}}{3}\right)$

The intermediate q-region $\left(0.12 \mathrm{~nm}^{-1} \leq \mathrm{q} \leq 0.21 \mathrm{~nm}^{-1}\right), \quad \mathrm{I}(\mathrm{q}) \sim \mathrm{q}^{-\alpha}$ (Power-law) and

$$
\text { The asymptotic region }\left(\mathrm{q} \geq 0.22 \mathrm{~nm}^{-1}\right), \quad \mathrm{I}(\mathrm{q})=\frac{I_{L}}{\left(\mathfrak{1}+\left(q-q_{0}\right)\right)^{m}}
$$

(Broad Peak region where $\mathrm{q}_{0}$ is related to peak position of scattered peak and $\xi$ is correlation length).

The crossover between regions between (i) and (ii) and between (ii) and (iii) designated as $\mathrm{q}^{*}$ and $\mathrm{q}^{* *}$ defined two other length scales,

$$
L^{*}=\frac{2 \Pi}{q^{*}} \quad(=50 \mathrm{~nm} \text { independent of concentration }) \text { and } L^{* *}=\frac{2 \Pi}{q^{* *}}
$$

( $=26$ to $22 \mathrm{~nm}$ decreasing with increase in DNA concentration). From statistical analysis of the structure factor data, we obtained the radius of gyration value, $R_{\mathrm{g}} \approx 40 \pm 3 \mathrm{~nm}$. Assuming, the strands to be rod-like,

the length of the strands $L$ can be determined from, $R_{g}^{2}=\frac{L^{2}}{12}$ which gives $\mathrm{L} \approx 140 \mathrm{~nm}$ which is quite realistic. In the high-q region a broad correlation peak was detected and the broad peak fitting analysis yielded correlation length, $\xi$ increasing from $0.50 \mathrm{~nm}$ to $3.30 \mathrm{~nm}$ and the inter strand spacingd decreasing from 15.6 to $9.8 \mathrm{~nm}$ as DNA content was increased from $1 \%$ to $3 \%$. A clear overlap concentration $\mathrm{c}^{*}=2 \%(\mathrm{w} / \mathrm{v})$ was found. DNA hydrogel was found to be consisting of hierarchy of structures with well-defined length scales that contribute uniquely to the scattering process. In summary; it has been shown that hierarchical structure of DNA hydrogels is replete with several self-organized assemblies, whose spatial extension is dependent on the biopolymer concentration.

\section{Acknowledgements}

PKP acknowledges University Grants Commission, Government of India for a Junior Research Fellowship. KR is very thankful to Department of Science and Technology, Government of India-Inspire Faculty Award. This work was supported by the Department of Science and Technology (DST), India.

\section{Conflict of interest}

The author declares no conflict of interest.

\section{References}

1. Horkay F, Basser PJ. Osmotic observations on chemically crosslinked DNA gels in physiological salt solutions. Biomacromolecules. 2004;5(1):232-237.

2. Feiyue Xu, Lei Xu, Qi Wang, et al. A three-dimensional dynamic coculture system enabling facile cell separation for chondrogenesis of mesenchymal stem cells. Biochemical Engineering Journal. 2015;103:68-76.

3. Jiang H, Pan V, Vivek S, et al. Programmable DNA hydrogels assembled from multidomain DNA strands. Chembiocheml. 2016;17(12):11561162 .

4. Papancea A, Valente AJ, Patachia S, et al. PVA-DNA cryogel membranes: characterization, swelling, and transport studies. Langmuir. 2008;24(1):273-279.

5. Cho YJ, Kim HY, Huang H, et al. Spectroscopic characterization of interstrand carbinolamine cross-links formed in the $5^{\circ}-\mathrm{CpG}-3^{\prime}$ sequence by the acrolein-derived $\gamma$-OH-1,N2-propano-2'-deoxyguanosine DNA Adduct. J Am Chem Soc. 2005;127(50):17686-17696.

6. Dutta S, Chowdhury G, Gates KS. Interstrand cross-links generated by abasic sites in duplex DNA. J Am Chem Soc. 2007;129(7):1852-1853.

7. Liedl T, Dietz H, Yurke B, et al. A pH-triggered, controlled trapping and release of quantum dots in a DNA-Switchable Hydrogel. Small. 2007;3(10):1688-1693.

8. Xing Y, Cheng E, Yang Y, et al. Self-assembled DNA hydrogels with designable thermal and enzymatic responsiveness. Adv Mater. 2011;23(9):1117-1121.

9. Moran MC, Miguel MG, Lindman B. DNA gel particles: particle preparation and release characteristics. Langmuir. 2007;23(12):6478-6481.

10. Lee CK, Shin SR, Lee SH, et al. DNA hydrogel fiber with self-entanglement prepared by using an ionic liquid. Angew Chem Int Ed Engl. 2008;47(13):2470-2474.

11. Orakdogen N, ErmanB, Okay O. Evidence of strain hardening in DNA gels. Macromolecules. 2010;43 (3):1530-1538.

12. Mason TG, Dhople A, Wirtz D. Linear viscoelastic moduli of concentrated DNA solutions. Macromolecules. 1998;31(11):3600-3603.

13. Murakami Y, Maeda M. DNA-responsive hydrogels that can shrink or swell. Biomacromolecules. 2005;6(6):2927-2929.

14. Liu Y, Jun Y, Steinberg V. Longest relaxation times of double-stranded and single-stranded DNA. Macromolecules. 2007;40(6):2172-2176.

15. Arfin N, Aswal VK, Kohlbrecher J, et al. Relaxation dynamics and structural changes in DNA soft gels. Polymer. 2015;65:175-182.

16. Costa D, Hansson P, Schneider S, et al. Interaction between covalent DNA gels and a cationic surfactant. Biomacromolecules. 2006;7(4):1090-1095. 
17. Okay O. DNA Hydrogels: New functional soft materials. Journal Of Polymer Science Part B: Polymer Physics. 2011;49:551-555.

18. He Y, Ye T, Su M, et al. Hierarchical self-assembly of DNA into symmetric supramolecular polyhedra. Nature. 2008;452(7184):198-201.

19. Li C, Jones AF, Dun AR, et al. Rapid formation of a supramolecular polypeptide-dna hydrogel for in situ three-dimensional multilayer bioprinting. Angew Chem Int Ed Engl. 2015;54(13):3957-3961.

20. Kohlbrecher J, Wagner W. The new SANS instrument at the swiss spallation source SINQ. J Appl Cryst. 2000;33:804-806.

21. Stauffer D, Aharony A. Introduction to Percolation Theory. 2nd ed. London, UK: Taylor and Francis; 1994.

22. Stauffer D, Coniglio A, Adam M. Gelation and critical phenomena In: Dušek K editor. polymer networks. Advances in Polymer Science. 1982;44:103-158.

23. De Arcangelis L, Del Gado E, Coniglio A. Complex dynamics in gelling systems. Eur Phys J E. 2002;9(3):277-282.
24. Debye P, Bueche AM. Scattering by an inhomogeneous solid. Journal of Applied Physics. 1949;20:518-525.

25. Mohanty B, Bohidar HB. Internal pressure fluctuations in coacervates and syneresis. EPL (Europhysics Letters). 2006;76(5):965-971.

26. Rawat K, Pathak J, Bohidar HB. Effect of persistence length on binding of DNA to polyions and overcharging of their intermolecular complexes in aqueous and in 1-methyl-3-octyl imidazolium chloride ionic liquid solutions. Phys Chem Chem Phys. 2013;15(29):12262-12273.

27. Mrevlishvili GM, Svintradze DV. Complex between triple helix of collagen and double helix of DNA in aqueous solution. Int J Biol Macromol. 2005;35(5):243-245.

28. Mohanty B, Aswal VK, Kohlbrecher J, et al. Length scale hierarchy in sol, gel, and coacervate phases of gelatin. J Polym Sci B Polym Phys. 2006;44(12):1653-1667.

29. Simmons BA, Irvin GC, Agarwal V, et al. Small angle neutron scattering study of microstructural transitions in a surfactant-based gel mesophase. Langmuir. 2002;18(3):624-632. 